\title{
M31 pixel lensing: Observations at the Loiano telescope
}

\author{
S. Calchi Novati* \\ on behalf of the PLAN collaboration ${ }^{b}$ \\ Dipartimento di Fisica "E. R. Caianiello", Università di Salerno, Via Ponte don Melillo, 84081 \\ Fisciano (SA), Italy and INFN, sez. di Napoli, Italy \\ *E-mail: novati@sa.infn.it \\ b http://plan.physics.unisa.it
}

\begin{abstract}
We discuss pixel lensing observations towards M31 carried out at the Loiano telescope. We have established a fully automatic pipeline for the detection and the characterization of microlensing events. We have evaluated the efficiency of the pipeline. We have estimated the expected signal by means of a Monte Carlo simulation. As a result we select 2 microlensing candidates. This is compatible with the expected M31 self-lensing signal. The small statistics of events at disposal does not still allow us, however, to draw definite conclusions on the content of compact halo objects.
\end{abstract}

Keywords: Microlensing; dark matter; M31

\section{Introduction}

The content of dark matter in galactic haloes in form of compact halo objects (MACHOs) is still an open issue ${ }^{1]}$ Following the original proposal of Paczyński, ${ }^{2}$ microlensing probed to be an efficient tool to carry out this research programme. The original target to explore the MACHO content of the halo of the Milky Way (MW) has been the Large Magellanic Cloud (LMC). Along this line of sight the more relevant results have been obtained by the MACHO ${ }^{3}$ EROS ${ }^{4}$ and $\mathrm{OGLE}^{\frac{5}{5}}$ collaborations. These observational programmes allowed to exclude MACHOs for a large range of masses as a possible dark matter component. Still, the results of the MACHO collaboration show that a sizeable mass fraction of the MW halo $(f \sim 20 \%)$ might be composed of compact halo objects of about $0.5 \mathrm{M}_{\odot}$. This outcome has been challenged by the EROS and the OGLE results. Once we accept that the reported candidate microlensing events are not to be attributed to intrinsic variable sources, a possible contamination of the MACHO lensing signal comes from self lensing, namely a lensing event where both the lens and the source belong to some luminous population. Indeed, the fact that the only remaining allowed mass range for MACHOs correspond to that of the stars that might also act as lenses may be indicative of some bias (because the characteristics of the microlensing events, in particular their duration, depend from the lens mass). On the other hand, if these events are really to be attributed to MACHOs in this mass range, a fortiori for such a sizeable fraction as that implied by the MACHO collaboration results, this might have some deeper astrophysical meaning. The LMC microlensing, together with microlensing towards the Galactic center used as a probe to constrain the inner Galactic structure, is the subject of the thorough review of Moniez: ${ }^{6}$

Beyond the LMC, the next suitable target for microlensing searches is M31. ${ }^{[79}$ It allows one to explore a different line of sight through the MW halo; we can fully map 
the M31 own dark matter halo (which is not possible for the MW one, this being perhaps the most severe limitation for LMC studies); finally, the inclination of M31 is expected to give rise to a characteristic signature in the spatial distribution of M31 halo events such to facilitate their identification against the contamination of selflensing events. However, because of the distance of M31, the sources for microlensing events are no longer resolved objects so that we enter the regime usually referred to as pixel lensing 10 Several observational campaigns have already been undertaken along this line of sight. As for the issue of the dark matter compact halo object content, the POINT-AGAPE collaboration claimed for an evidence of a signal in the same mass range indicated by the MACHO LMC analysis.11 This outcome, however, has been challenged by the MEGA collaboration who, using the same set of data, concluded that their detected signal is compatible with the expected selflensing rate. ${ }^{12}$ In fact, especially if one does not move far enough from the M31 center, the contamination of self lensing, still difficult to be exactly quantified, is in any case expected to be quite large with respect to MACHO lensing. Pixel lensing towards M31 is the subject of the review of Calchi Novat13 (we also recall the recent analysis of Tsapras et al.,14 where a new analysis of the POINT-AGAPE data set is presented together with a comparison of the results of different pipelines). In the present contribution we report on the ongoing pixel lensing campaign towards M31 carried out by the PLAN collaboration.

\section{Microlensing candidate events}

Our observational programme started in 2006 using the $152 \mathrm{~cm}$ Loiano telescope at the Osservatorio Astronomico di Bolognal. We have been monitoring two fields of $13^{\prime} \times 12.6^{\prime}$ each around the M31 center (Fig. 1a) to probe both self lensing and MACHO lensing! 15 We observe for consecutive (and full) nights in order to be able to properly sample and characterize the microlensing events and get to a sufficient signal to noise ratio. Since 2008 we are also using the $150 \mathrm{~cm}$ TT1 telescope at the Osservatorio Astronomico di Capodimonte (Napoli)b. The results presented below, out of the 2007 observational campaign, are detailed in Calchi Novati et al 16

We select microlensing candidates on the basis of a series of, fully automatised, criteria, among which an analysis on the shape and the sampling of the flux variations, to exclude intrinsic variable sources, and an analysis of the PSF at maximum amplification, to exclude fake events (cosmic rays, bad pixels ...). Furthermore, we limit our search to bright events with a large enough signal to noise ratio. As a result we select two microlensing candidate, OAB-N1 and OAB-N2. Neither of them, however, can be looked at as a fully convincing candidate. In particular, we lack data points along the descent of OAB-N2 (Fig. 1b) so that we can not probe the expected symmetric shape characteristic of microlensing events, although the excellent agreement, on the raising part, with a Paczyński shape, together with its

attp://www.bo.astro.it/loiano/index.html

bttp://www.na.astro.it/tt1/ 


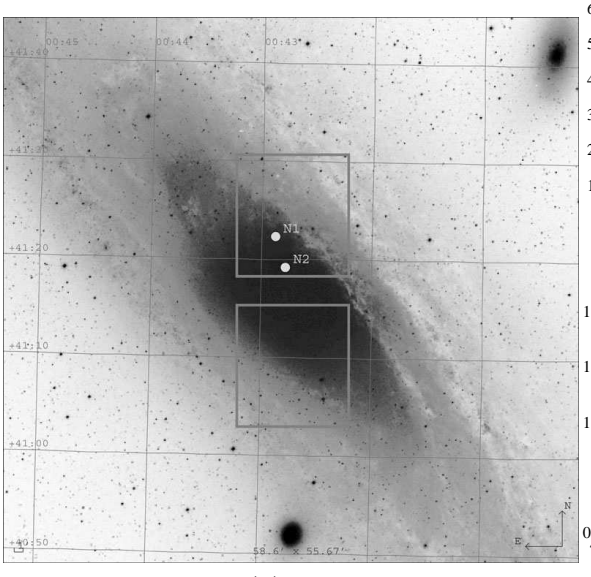

(a)
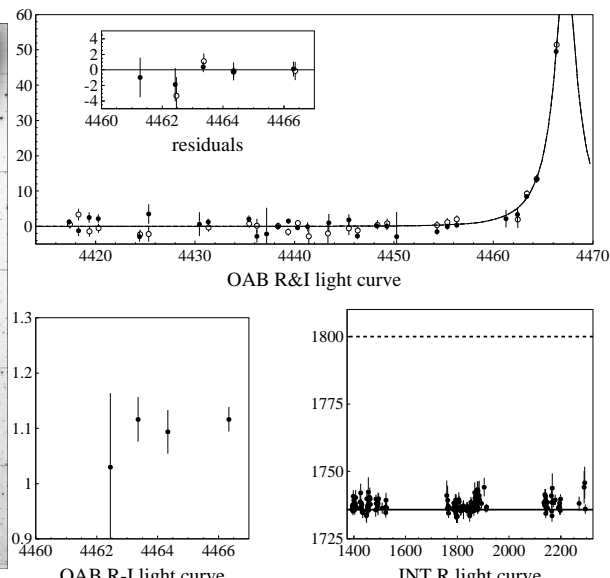

INT R light curve

(b)

Fig. 1. (a) Superimposed on a wide field image of M31 we show the monitored fields of view and the position of the two candidate microlensing events. (b) For the OAB-N2 microlensing candidate we show the light curve with the best Paczyński fit, the residual to the Paczyński best fit, the achromaticity test light curve along the bump and the (flat) extension along the INT data (the dashed line indicates the flux variation corresponding to that observed along the OAB data). The units on the $x$ axes are time in days (JD-2450000.0). The ordinate axes units are magnitude for the bottom left panel, and flux in $\mathrm{ADU} \mathrm{s}{ }^{-1}$ per superpixel for the remaining panels.

characteristics (duration and brightness at maximum amplification) are strongly suggestive of the truly microlensing origin of this flux variation. In addition to the already probed flatness of the OAB-N2 light curve extension along three years of POINT-AGAPE data, the preliminar analysis of the data of the 2008 and 2009 observational campaigns at Loiano also confirms the unicity of the OAB-N2 flux variation.

Next step in the analysis, we have to estimate the expected signal. The number and the characteristics (among which the most relevant, for a first order analysis, are the position and the duration) both for M31 self lensing and MACHO lensing (for which about $1 / 3$ of the events are expected, for given halo mass fraction and MACHO mass, to belong to the MW halo). To this purpose we carry out a full Monte Carlo simulation of the experiment where we model M31, the microlensing amplification and we take into account the observational set up. A relevant issue of the above analysis is the modeling of the M31 luminous components responsible for the expected self-lensing signal, to be compared with MACHO lensing: in particular there is still a debate about the exact value of the overall mass to be attributed to the stellar lenses, an important parameter of the model as it is directly proportional to the number of the expected self-lensing events. Finally, to properly compare to the output of the selection pipeline, we simulate the microlensing events selected within the Monte Carlo simulation in the raw data and run from scratch the full selection process so to reliably assess the efficiency of the pipeline. 
Microlensing events towards M31 are expected to be short duration (usually shorter than 10 days) flux variations, with, for MACHO lensing, a broader spatial distribution than for self lensing. The expected rate we evaluate for our campaign is small. Somewhat smaller than 1 event for self lensing, and about the same for MACHO lensing (for istance, we expect 2 events for a full halo of $0.1 \mathrm{M}_{\odot}$ compact objects). The detected events are therefore compatible with the expected self-lensing rate, however our available statistics is still too small to draw definite conclusions on the dark matter content in the form of compact halo objects towards M31. Currently, we are completing the analysis of the 2008 and 2009 campaigns.

\section{Discussion}

Pixel lensing searches towards M31 suffer the limitation given by the small expected rate of events (overall, up to now, the detection of about 20-30 candidate events has been reported). This makes particularly troublesome the disentanglement among self-lensing and MACHO lensing events. Analogously to the case of the Galactic center, the self-lensing signal is an invaluable tool to probe the stellar content of M31, and in fact the ANGSTROM collaboration is focused on this aspect!17 On the other hand, for a better understanding of MACHO lensing two main paths may be followed. First, to increase the statistics of the detected events. Besides carrying out long enough observational campaigns, this means to look for fainter flux variations (where the risk of contamination by intrinsic variable sources is however much larger) and, this is essential to probe MACHO lensing against self lensing, to map the full area of M31. In this respect, the PAndromeda project is expected to represent a real "second-generation" M31 pixel lensing observational programme. Second, to look for a better light curve characterization and astrophysical understanding of single events. A first example has been the thorough analysis of the POINT-AGAPE PA-N1 candidate event ${ }^{[18}$ A second case has been the PA-S3/GL1 candidate event, $\frac{1920}{20}$ throughly characterized by the WeCAPP collaboration, $\frac{21}{1}$ for which it has been shown, in particular on the basis of its large amplification at maximum and on a detailed study of the finite size source effect, that MACHO lensing is more likely than self lensing. As a PLAN collaboration we intend to pursue both these objectives. First, by completing the analysis of the 2008 and 2009 observational campaigns. Second, by carrying out a more detailed analysis, making use also of some new data, of the OAB-N2 candidate event. ${ }^{22}$ A fundamental aspect of the above mentioned analyses is the merging of different data sets. This is relevant both because it naturally strengthens the microlensing interpretation of the given flux variation and because of the increase in the sampling, often essential for a better characterization of the event. This is indeed a usual approach for Galactic bulge

${ }^{\mathrm{c}}$ S. Seitz, talk given at the 13th Microlensing Workshop, 2009, Paris. This observational programme is planned to make use of the $1.8 \mathrm{~m}$ PS1 telescope with a huge field of view of 6.4 sqdeg so to cover in a single shot all of the M31 field. M31 is expected to be monitored with a cadence of nightly exposures for about 10 weeks per season. The first-year campaign has started in fall 2009. 
light curves, but it clearly shows the path to be followed also for M31 pixel lensing event analyses. In this respect, the attempt of the ANGSTROM collaboration $\frac{17}{17}$ to establish a real time analysis of M31 pixel lensing flux variations, the Angstrom Project Alert System ${ }^{23}$ (APAS), is extremely relevant. This is indeed a first important step towards a full survey-follow up strategy. (This is once more a usual approach for Galactic bulge analyses that would certainly greatly help to improve our understanding of the M31 lensing signal.) Finally, we recall that microlensing is already currently used as a powerful tool for the research of extra-solar planets towards the Galactic bulge. 24 The coordination among different data sets and observational campaigns are essential ingredients for the, extremely challenging, purpose of the research of pixel lensing planet signatures in M31.25

\section{References}

1. R. Massey, T. Kitching and J. Richard, arXiv:1001.1739 (2010)

2. B. Paczyński, ApJ 304, 1 (1986).

3. C. Alcock, et al., ApJ 542, 281 (2000).

4. P. Tisserand, et al., A\&A 469, 387 (2007).

5. Ł. Wyrzykowski, et al., MNRAS 397, 1228 (2009).

6. M. Moniez, GRG in press (2010).

7. A. P. S. Crotts, ApJ 399, L43 (1992).

8. P. Baillon, A. Bouquet, Y. Giraud-Heraud and J. Kaplan, A $\& A$ 277, 1 (1993).

9. P. Jetzer, $A \& A$ 286, 426 (1994).

10. A. Gould, ApJ 470, p. 201 (1996).

11. S. Calchi Novati, et al., $A \& \mathcal{E} A$ 443, 911 (2005).

12. J. T. A. de Jong, et al., $A \& A$ 446, 855 (2006).

13. S. Calchi Novati, GRG in press, arXiv:0912.2667 (2009).

14. Y. Tsapras, et al., MNRAS in press, arXiv:0912.2696, (2009).

15. S. Calchi Novati, et al., $A \mathscr{E} A$ 469, 115 (2007).

16. S. Calchi Novati, et al., ApJ 695, 442 (2009).

17. E. Kerins, et al., MNRAS 365, 1099 (2006).

18. M. Aurière, et al., ApJ 553, L137 (2001).

19. S. Paulin-Henriksson, et al., A $\& A$ 405, 15 (2003).

20. A. Riffeser, et al. ApJ 599, L17 (2003).

21. A. Riffeser, S. Seitz and R. Bender, ApJ 684, 1093 (2008).

22. S. Calchi Novati, et al., in preparation (2010).

23. M. J. Darnley, et al. ApJ 661, L45 (2007).

24. M. Dominik, $G R G$ in press (2010).

25. G. Ingrosso, et al., MNRAS 399, 219 (2009). 